\title{
Valvular Heart Disease-Related Feed-Forward Loop Network Reveals Some Biological Features for Disease Development
}

\author{
Feng YANG ${ }^{1,2}$, Ke LIU ${ }^{3}$, Wei ZENG ${ }^{1,2}$, Guang-bin WANG ${ }^{1,2}$, \\ Zheng-wen $\mathrm{LI}^{1,2}$, Ke-li HUANG,a, ${ }^{2, *}$ and Ni-ni RAO ${ }^{1,2,3, b,{ }^{*}}$ \\ ${ }^{1}$ Center for Information Biology, University of Electronic Science and Technology of \\ China, Chengdu, China \\ ${ }^{2} S$ chool of Life Science and Technology, University of Electronic Science and \\ Technology of China, Chengdu, China \\ ${ }^{3}$ Subsidiary hospital, University of Electronic Science and Technology of China, \\ Chengdu, China \\ ${ }^{4}$ Guangdong Electronic Information Engineering Research Institute, University of \\ Electronic Science and Technology of China, Dongguan, Guangdong \\ ahuangkeli1968@163.com, ${ }^{b}$ raonn@uestc.edu.cn, \\ ${ }^{*}$ Corresponding author
}

Keywords: Valvular heart disease (VHD), MicroRNA (miRNA), Transcription factor (TF), Gene, Feed-forward loop network (FFL).

\begin{abstract}
This study is to uncover VHD-related important biological features for understanding molecular of this disease. The system analysis strategy is applied to generate the first VHD-related FFL network. Then, the network topology is mapped into the biological space to refine the biological features of VHD. 4 miRNA, 4 TF and 34 gene hubs are identified and confirmed to play important roles in the occurrence and development of VHD. The signal pathways mediated by miRNA hubs are also predicted, where the processes that MAPK pathway mediated by has-let-7c induces VHD are explained. This study provides some new computational evidences for the understanding of VHD molecular mechanism, and also has important implications in terms of improving current therapeutic strategies of VHD.
\end{abstract}

\section{Introduction}

The miRNAs, TFs and genes interact to form a network system, in which miRNAs and TFs are major regulators and they are closely linked to regulate common target genes and to participate in many important biological processes, including cell proliferation, differentiation, apoptosis, etc.

Many complex diseases are associated with disorders of TFs and miRNAs [1], which will cause that the biological regulatory system of an organism appears to adjust abnormal, the internal homeostasis of system is broken and the symptoms of the disease in vitro are shown. So far, a lot of efforts have been made to construct the regulatory network associated with an objective disease based on interaction relationships among miRNA, TF and their target genes, such as the ovarian cancer [2], the glioblastoma multiforme [3], the osteosarcomas [4], etc. The existing studies showed that: (i) the analysis of complex diseases can benefit from systems biological approaches; (ii) FFLs are conservative and have basic biological functions in the complex regulatory relationships among miRNAs, TFs and genes, meanwhile it can be used as a basic unit to construct a regulatory network.

VHD is the function or structural abnormality of one or more valve structures due to 
multiple causes, such as inflammation, mucus degeneration, degenerative changes, congenital malformations, ischemic necrosis, trauma, etc. It is a major cause of morbidity and premature death from cardiovascular diseases and still remains highly prevalent. Although this disease has been verified to have various genomic alterations [5-7], its molecular mechanism is not fully understood yet. Researches on VHD-related network consisting of miRNAs, TFs and their targets and the control mechanisms are still in the blank state at present. To end this, this paper applied a system analysis strategy to construct FFL network related to VHD. Through analyzing the network topology and mapping it into biological space, some important biological features associated with the development of VHD were found. This work can provide the theoretical evidences for understanding interaction mechanisms and regulation patterns among miRNAs, TFs and target genes in VHD development.

\section{Materials and Methods}

\section{MiRNAs Related to VHD}

VHD-related miRNAs were collected through performing a comprehensive literature searching and reviewing. First, a group of relevant articles were compiled from Google scholar (http://scholar.google.com.hk/) using the search phrase "valvular heart disease" and from PubMed (http://www.ncbi.nlm.nih.gov/pubmed/) using the search phrase "valvular heart disease\&miRNA". After carefully analyzing the related literatures, 15 VHD-related miRNAs that have been experimentally verified and met the requirements of this study were ultimately selected. The information of 15 VHD-related miRNAs is shown in Table 1.

Table 1. The information of 15 VHD-related miRNAs

\begin{tabular}{|c|c|c|c|c|c|c|c|c|}
\hline No & Symbol & source & No. & Symbol & source & No. & Symbol & source \\
\hline 1 & $\begin{array}{c}\text { hsa-miR-3 } \\
\text { 0b }\end{array}$ & [8] & 6 & hsa-miR-125b & [12] & 11 & $\begin{array}{c}\text { hsa-miR-9 } \\
\text { 9b }\end{array}$ & {$[12]$} \\
\hline 2 & $\begin{array}{c}\text { hsa-miR-1 } \\
41\end{array}$ & [9] & 7 & hsa-miR-127 & [12] & 12 & $\begin{array}{c}\text { hsa-miR-3 } \\
28\end{array}$ & [12] \\
\hline 3 & $\begin{array}{c}\text { hsa-miR-2 } \\
1\end{array}$ & [10] & 8 & $\begin{array}{c}\text { hsa-miR-199a-3 } \\
\text { p }\end{array}$ & [12] & 13 & $\begin{array}{c}\text { hsa-miR-7 } \\
44\end{array}$ & [12] \\
\hline 4 & $\begin{array}{c}\text { hsa-miR-2 } \\
3 a\end{array}$ & [11] & 9 & hsa-miR-204 & [12] & 14 & $\begin{array}{c}\text { hsa-miR-2 } \\
6 \mathrm{a}\end{array}$ & [13] \\
\hline 5 & hsa-let-7c & [12] & 10 & hsa-miR-320 & [12] & 15 & $\begin{array}{c}\text { hsa-miR-1 } \\
95\end{array}$ & {$[13]$} \\
\hline
\end{tabular}

\section{Predicting Relationships of miRNA-gene, TF-gene/miRNA and miRNA-TF}

In order to minimize the false positives and to increase the reliability of prediction results, we predicted the target genes of each VHD-related miRNA using TargetScan [14], miRanda [15] and PicTar [16] databases. Only those relationships simultaneously appearing in the three databases were retained in this study.

To predict the regulatory relationships between TFs-genes and TFs-miRNAs, we 
first downloaded the defined promoter region (-2000/+2000 around CDS) of VHD-related miRNAs and their target genes. Then, we performed a binding sites search using the Match ${ }^{\mathrm{TM}}$ [17] software that is integrated in TRANSFAC Professional [18]. For the purpose of this study, we used the pre-calculated cut-offs to minimize the false positive matches and create a high-quality matrix. To restrict the search, we set a core score of 1.00 and a matrix score of 0.95 , and selected TFs associated with the human genome only. Finally, we established the relationships of miRNA-TF through their common target genes.

\section{Constructing VHD-related FFL Network}

The FFLs have mainly the three types: TF-FFL, miRNA-FFL and composite-FFL [3]. In a TF-FFL motif, a TF regulates both miRNA and gene and a miRNA represses its target gene. In a miRNA-FFL motif, a miRNA represses both TF and its target gene, as well as a TF regulates a gene. In a composite-FFL motif, a TF regulates both miRNA and gene, as well as a miRNA represses both TF and gene.

We first tallied all the relationship pairs among miRNAs, TFs and genes above. Then, according to the tallied results, we constructed the TF-FFL motifs, the miRNA-FFL motifs and the composite-FFL motifs. Next, we calculated the P-values of miRNA-TF pairs in each type of the FFLs using the cumulative hypergeometric test shown in the equation (1):

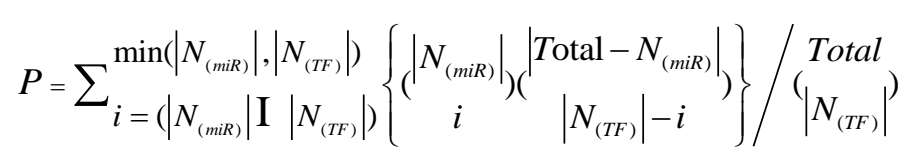

where $N_{\text {(miRNA) }}$ is the number of genes targeted by a given miRNA, $N_{\text {(TF) }}$ is the number of genes regulated by a given TF, and Total is the number of common genes between all human genes targeted by human miRNAs and all human genes regulated by all human TFs. Furthermore, the multiple test with false discovery rate (FDR) of less than 0.05 [19] is performed. Only those FFLs with the miRNA-TF pairs of $P<0.05$ in each type of FFLs were screened. Finally, through blending the three kinds of FFLs with significant miRNA-TF pairs, the VHD-related FFL network is constructed.

\section{Mapping Network Topology into Biological Space for Critical Biological Features}

A biological network is with scale-free, that is, the degree distribution of nodes obeys power-law distribution, indicating that most nodes had a low degree, while only a small portion of nodes had a high degree. Therefore, we can observe only a few of miRNAs, genes and TFs to exhibit a high degree in a biological network. These critical molecules act as hubs that might play important roles in maintaining the overall connectivity of a disease-related network [20].

According to the above views, we firstly count the degree of each node on the VHD-related FFL network. Then, we rank the nodes according to their degree numbers and types. Next, we extracted miRNAs and TFs arranged in the top 25\% of the degrees and genes arranged in the top 5\% of the degrees as the hubs, and run the functional annotation clustering Tools GO and KEGG to obtain the critical biological features of the hubs. Finally, we analyze the roles of key nodes in the cell for understand important molecular mechanism of the VHD disease from the activation of biological molecules, to opening of the corresponding biological signaling pathways, and to influence of specific biological process behavior on diseases. 


\section{Results}

\section{The Topology Characteristics of Constructed VHD-related FFL Network}

The topology characteristics of VHD-related FFL network constructed are shown in Table 2.

Table 2. The topology characteristics of VHD-related FFL network

\begin{tabular}{|c|c|c|c|c|c|c|c|c|}
\hline & FFLs & Gene & miRNA & TF & $\begin{array}{l}\text { miRNA- } \\
\text { gene }\end{array}$ & $\begin{array}{l}\text { miRNA- } \\
\text { TF }\end{array}$ & $\begin{array}{l}\text { TF- } \\
\text { gene }\end{array}$ & $\begin{array}{l}\text { TF- } \\
\text { miRNA }\end{array}$ \\
\hline Number & 1016 & 691 & 12 & 23 & 845 & 21 & 893 & 24 \\
\hline
\end{tabular}

The degree distribution curve of genes is shown in Fig. 1, which obeys the power law distribution. Because the numbers of miRNAs and TFs in this network are too low, their degree distribution curves lack statistical significances.

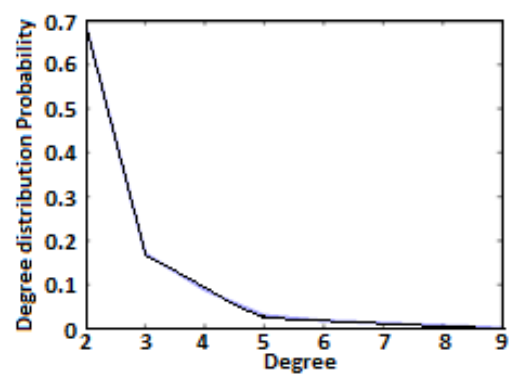

Fig. 1. The degree distribution curve of genes in VHD-related FFL network.

\section{The Critical Hubs and Their Biological Features}

Those miRNAs, TFs and genes that their degrees are ranked in the top 25\% and 5\% respectively are identified as the hubs. The results are shown in Table 3, including 4 miRNAs (hsa-let-7c, hsa-miR-23a, hsa-miR-26a and hsa-miR-195), 4 TFs (Nkx2-5, Oct-1, FOXD3 and HNF-3beta) and 34 genes.

Table 3. The identified hubs according to the degree ranking

\begin{tabular}{|c|c|c|c|c|c|l|}
\hline miRNA Hubs & TF Hubs & \multicolumn{5}{|c|}{ Gene Hubs } \\
\hline hsa-let-7c & Nkx2-5 & QKI & KPNA4 & CAB39 & WHSC1 & FUT4 \\
\hline hsa-miR-23a & Oct-1 & CDC25A & CXCL12 & DCBLD2 & ATP2A2 & ELF2 \\
\hline hsa-miR-26a & FOXD3 & PLAGL1 & GPRC5B & LYPLA2 & PPARGC1A & RBM24 \\
\hline hsa-miR-195 & HNF-3beta & POM121 & ANKHD1 & DMTF1 & PRDM1 & SOX5 \\
\hline & & CPEB3 & CAMTA1 & PPP1R15B & STK35 & LRIG1 \\
\hline & & HAS2 & ATXN1 & SLC1A1 & RAP2C & CCND1 \\
\hline & & MTPN & PURB & TNKS2 & SLC7A1 & \\
\hline
\end{tabular}

We predicted 140 target genes of hsa-let-7c and respectively obtained 116, 100 and 122 terms of biological processes, cellular components and molecular functions through GO analysis. In summary, the enriched biological processes mainly included protein kinase cascade $(29 / 116,25 \%)$, molecular signaling pathway $(24 / 116,20.7 \%)$, protein modification $(12 / 116,10.3 \%)$, and metabolism regulation $(28 / 116,24.1 \%)$; The enriched cellular components mainly included proteinaceous extracellular matrix $(7 / 100,7 \%)$ and plasma membrane part $(30 / 100,30 \%)$; the enriched molecular function terms have mainly protein kinase activity (12/122,9.8\%). For hsa-miR-23a, we predicted its 119 target genes and respectively obtained 106, 78 and 95 terms of biological processes, cellular components and molecular functions through GO analysis. In summary, the enriched biological processes mainly included protein kinase cascade $(20 / 106,18.9 \%)$, regulation of transcription $(17 / 106,16.0 \%)$, and regulation of metabolic process $(39 / 106,36.8 \%)$; the enriched cellular components are mainly cell junction $(9 / 78,11.5 \%)$, etc. the enriched molecular function terms have mainly protein 
kinase activity $(13 / 95,13.7 \%)$ and transcription regulator activity $(18 / 95,18.9 \%)$. For hsa-miR-26a, we predicted its 124 target genes and respectively obtained 98, 84 and 96 terms of biological processes, cellular components and molecular functions through GO analysis. In summary, the enriched biological processes are mainly involved in intracellular signaling cascade $(22 / 98,22.4 \%)$, protein amino acid phosphorylation $(12 / 98,12.2 \%)$ and metabolic process $(21 / 98,21.4 \%)$; the enriched cellular components are mainly cytoskeleton $(17 / 84,20.2 \%)$; The molecular function terms have mainly protein kinase activity $(11 / 96,11.5 \%)$ and molecular binding (46/96, 47.9\%). For hsa-miR-195, we predicted its 120 target genes (Table S4) and respectively obtained 100, 81 and 94 terms of biological processes, cellular components and molecular functions through GO analysis. In summary, the enriched biological processes mainly are involved in regulation of cell cycle $(17 / 100,17 \%)$, protein catabolic process $(11 / 100,11 \%)$ and metabolic process $(25 / 100,25 \%)$; The enriched cellular components include plasma membrane $(34 / 81,42.0 \%)$ and cytoplasmic vesicle $(10 / 81,12.3 \%)$; The molecular function terms have mainly protein kinase activity $(11 / 94,11.7 \%)$ and phosphatase activity $(6 / 94,6.4 \%)$.

The predicted signal pathways mediated by hsa-let-7c, hsa-miR-23a, hsa-miR-26a and hsa-miR-195 using KEEG analysis respectively, are shown in Table 4.

Table 4 . The predicted signal pathways mediated by miRNA hubs

\begin{tabular}{|c|l|c|c|}
\hline miRNA Symbol & The mediated signal pathway & $P$ Value & $\begin{array}{c}\text { Involved gene count } \\
\text { ( proportion) }\end{array}$ \\
\hline \multirow{4}{*}{ Hsa-let-7c } & MAPK signaling pathway & $1.10 \mathrm{E}-02$ & $8(16.7 \%)$ \\
\cline { 2 - 4 } & Pancreatic cancer & $2.80 \mathrm{E}-02$ & $4(8.3 \%)$ \\
\cline { 2 - 4 } & Axon guidance & $3.00 \mathrm{E}-02$ & $5(10.4 \%)$ \\
\cline { 2 - 4 } & Chronic myeloid leukemia & $3.10 \mathrm{E}-02$ & $4(8.3 \%)$ \\
\hline \multirow{2}{*}{ Hsa-miR-23a } & Heparan sulfate biosynthesis & $1.70 \mathrm{E}-02$ & $3(7.5 \%)$ \\
\hline \multirow{5}{*}{ Hsa-miR-26a } & mTOR signaling pathway & $6.10 \mathrm{E}-03$ & $4(10.5 \%)$ \\
\cline { 2 - 4 } & Cell cycle & $1.30 \mathrm{E}-03$ & $7(13.7 \%)$ \\
\cline { 2 - 4 } & Wnt signaling pathway & $3.40 \mathrm{E}-03$ & $7(13.7 \%)$ \\
\cline { 2 - 4 } & Pathways in cancer & $4.10 \mathrm{E}-03$ & $10(19.6 \%)$ \\
\cline { 2 - 4 } & Pancreatic cancer & $5.20 \mathrm{E}-03$ & $5(9.8 \%)$ \\
\cline { 2 - 4 } & Chronic myeloid leukemia & $6.00 \mathrm{E}-03$ & $5(9.8 \%)$ \\
\cline { 2 - 4 } & Axon guidance & $3.70 \mathrm{E}-02$ & $5(9.8 \%)$ \\
\cline { 2 - 4 } & Focal adhesion & $4.60 \mathrm{E}-02$ & $6(11.8 \%)$ \\
\cline { 2 - 4 } & Colorectal cancer & $4.90 \mathrm{E}-02$ & $4(7.8 \%)$ \\
\hline
\end{tabular}

By performing GO analysis of 34 critical genes, we obtained 30, 22 and 27 terms of biological processes, cellular components and molecular functions. respectively. In summary, the enriched biological processes are mainly involved in cell chemical property balance $(6 / 30,20 \%)$ and metabolic regulation $(15 / 30,50 \%)$; the important cellular component is nuclear membrane $(3 / 22,13.6 \%)$; the terms of molecular functions have mainly transcriptional regulator activity $(8 / 27,29.6 \%)$. No signal pathway is enriched on KEGG for 34 gene hubs.

At present, some scholars have used the experimental methods to explore the pathogenesis of VHD disease at molecular level, including: extracellular matrix remodeling [21], cell gap junction [22], cell metabolism regulation [23] and abnormal cell signals, such as TGF- $\beta$ [24], Wnt [25], mTOR [26], MAPK [27], cell cycle signal 
[28], etc. Thus, the biological features of the identified 4 miRNA and 34 gene hubs at biological processes, cellular components and molecular functions have high matching degree with the verified pathological factors above. This illustrates that these hubs might play a major role in the occurrence and development of VHD disease.

\section{The Roles of MAPK Signal Pathways Mediated by Hsa-let-7c in VHD Development}

Using the Go analysis results above and the existing evidences for the biological functions of MAPK pathway, we summarized the process that hsa-let-7c mediates MAPK signal pathway to induce VHD, as shown in Fig. 2. Once the receptor proteins on cell membrane like CACNA1E, ACVR1B and TNFR bind the relevant cell external active proteins, MAPK signal is activated. On the one hand, the active CACNA1E receptor can jointly activate a series of kinases and then acts on NRAS, which is an important Ras protein in MAPK signaling pathway and can connect the upstream pathway Ras-GAPs and the downstream pathway Rho/Rac. The active Ras protein can indirectly activate ERK, an extracellular signal-regulated kinase. MAPK/ERK pathway is a classical signal transduction pathway and it can mediate the cell proliferation and differentiation. On the other hand, the membrane receptor ACVR1B can trigger the kinases in connection with P38 signaling pathway, meanwhile TNFR acts on CASP, an enzyme related to apoptosis. CASP further activates PAK1, an important transfer enzyme, which can initiate JNK signaling enzymes as well as NLK and MAP3K3. P38 and JNK alternately motivate p53 signal, which is related to inflammatory reaction and adjusts apoptosis of cells. NLK and MAP3K3 further activate Wnt signal pathway, which mediates actin reorganization and remodels cytoskeleton via the complex cross-talking between signals. The existing study found that P38, JNK, ERK and P53 pathways change physiological structure of cardiac valve and take part in VHD development through affecting skeleton, fibrosis, mitosis and apoptosis of cardiac muscle cells [21]. This supports our explanation.

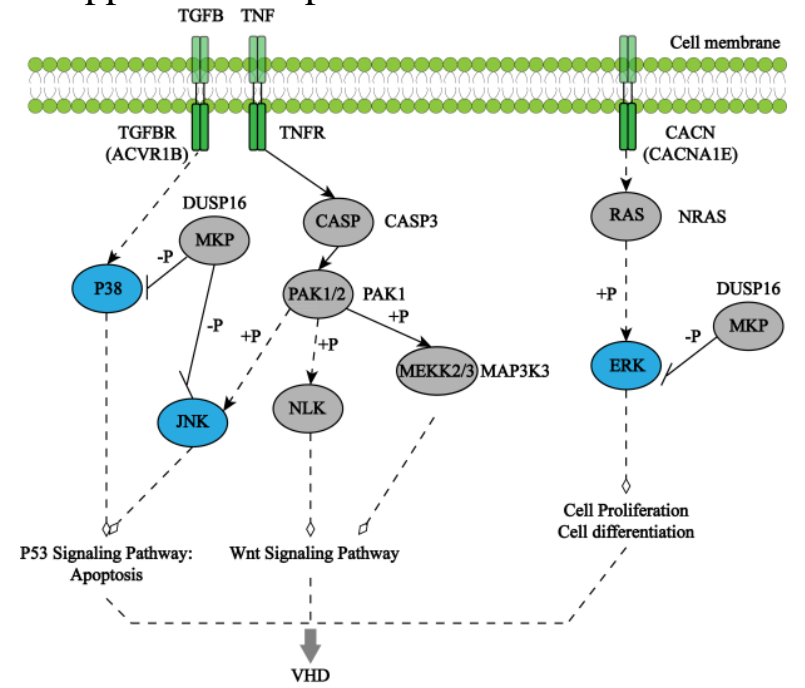

Fig. 2. The process that hsa-let-7cmediates MAPK signal pathway to induce VHD.

\section{Conclusions}

This study represents the first attempt to investigate FFL networks in the pathogenesis of VHD. 4 miRNA (hsa-miR-23a, hsa-miR-195, hsa-miR-26a and hsa-let-7c), 4 TF (Nkx2-5, Oct-1, FOXD3 and HNF-3beta) and 34 gene hubs are identified through the analysis of the network topology. Since there are high matching degree between the 
biological features of VHD-related hubs and the verified VHD pathological factors, these hubs might play an important role in VHD development. We predicted VHD-related signal pathways mediated by miRNA hubs, in which the roles of MAPK signal pathway mediated by hsa-let-7c in VHD development are analyzed. This study provides some new computational evidences for the understanding of VHD molecular mechanism.

\section{Acknowledgments}

This work was supported by National Natural Science Foundation of China (Grant No. 81171411), Sichuan Science and Technology Support Program (Grant No. 2015SZ0191) and Key project of Natural Science Foundation of Guangdong province (2016A030311040).

\section{References}

[1] K. I. Goh, M. E. Cusick, D. Valle, et al., The human disease network, Proc. Natl. Acad. Sci. 104 (2007) 8685-8690.

[2] S. Schmeier, U. Schaefer, M. Essack, V. B. Bajic, Network analysis of microRNAs and their regulation in human ovarian cancer, BMC Systems Biology 5 (2011) 1-9.

[3] J. Sun, X. Gong, B. Purow, et al., Uncovering microRNA and transcription factor mediated regulatory networks in Glioblastoma, PLoS Comput. Biol. 8 (2012) 1-14.

[4] K. Poos, J. Smida, M. Nathrath, et al., How microRNA and transcription factor co-regulatory networks affect Osteosarcoma cell proliferation, PLoS Comput. Biol. 9 (2013) 262-279.

[5] J. Xiao, D. Liang, Y. Zhang, Y. Liu, H. Zhang, Y. Liu, L. Li, X. Liang, Y. Sun, Y. H. Chen, MicroRNA expression signature in atrial fibrillation with mitral stenosis, Physiol Genom 43 (2011) 655-664.

[6] N. Cooley, M. J. Cowley, R. C. Lin, S. Marasco, C. Wong, D. M. Kaye, A. M. Dart, E. A. Woodcock, Influence of atrial fibrillation on microRNA expression profiles in left and right atria from patients with valvular heart disease, Physiol Genom. 44 (2012) 211-219.

[7] G. Lamirault, N. Gaborit, M. N. Le, C. Chevalier, G. Lande, S. Demolombe, D. Escande, S. Nattel, J. J. Leger, M. Steenman, Gene expression profile associated with chronicatrial fibrillation and underlying valvular heart disease in man, J. Mol. Cell Cardiol. 40 (2006) 173-184.

[8] M. Zhang, X. Liu, X. Zhang, et al., MicroRNA-30b is a multifunctional regulator of aortic valve interstitial cells, The Journal of Thoracic and Cardiovascular Surger 147 (2013) 1073-1080.

[9] B.Yanagawa, F. Lovren, Y. Pan, et al., miRNA-141 is a novel regulator of BMP-2-mediated calcification in aortic stenosis, The Journal of thoracic and cardiovascular surgery 144 (2012) 256-262.

[10] A. V. Villar, R. García, D. Merino, M. Llano, M. Cobo, C. Montalvo, et al., Myocardial and circulating levels of microRNA-21 reflect left ventricular fibrosis in aortic stenosis patients, Int. J. Cardiol. 167(2013) 2875-2881. 
[11] A. K. Lagendijk, M. J. Goumans, S. B. Burkhard, et al., MicroRNA-23 restricts cardiac valve formation by inhibiting Has2 and extracellular hyaluronic acid production, Circulation research 109(2011) 649-657.

[12] C. Vacchisuzzi, F. Hahne, P. Scheubel, M. Marcellin, V. Dubost, et al., Heart structure-specific transcriptomic atlas reveals conserved microRNA-mRNA interactions, PLoS One 8 (2013) 1-13.

[13] V. Nigam, H. H. Sievers, B. C. Jensen, et al., Altered microRNAs in bicuspid aortic valve: a comparison between stenotic and insufficient valves, Journal of Heart Valve Disease 19 (2010) 459-465.

[14] A. Grimson, K. H. Farh, W. K. Johnston, et al., MicroRNA targeting specificity in mammals: determinants beyond seed pairing, Molecular Cell 27 (2007) 91-105.

[15] D. Betel, M. Wilson, A. Gabow, D. S. Marks, C. Sander, The microRNA.org resource: targets and expression, Nucleic Acids Res. 36 suppl_1 (2008) D149-D153.

[16] A. Krek, D. Grun, M. N. Poy, et al., Combinatorial microRNA target predictions, Nature Genetics 37 (2005) 495-500.

[17] A. E. Kel, E. Gossling, I. Reuter, et al., MATCH: A tool for searching transcription factor binding sites in DNA sequences, Nucleic Acids Res. 31 (2003) 3576-35769.

[18] T. Heinemeyer, E. Wingender, I. Reuter, et al., Databases on transcriptional regulation: TRANSFAC, TRRD and COMPEL, Nucleic Acids Res. 26 (1998) 362-367.

[19] Y. Benjamini, Y. Hochberg, Controlling the false discovery rate-a practical and powerful approach to multiple testing, Journal of the Royal Statistical Society-Series 57 (1995) 289-300.

[20] A. L. Barabasi, Z. N. Oltvai, Network biology: understanding the cell's functional organization, Nat. Rev. Genet. 5(2004) 101-113.

[21] O. Fondard, D. Detaint, B. Iung, et al., Extracellular matrix remodelling in human aortic valve disease: the role of matrix metalloproteinases and their tissue inhibitors, European heart journal 26 (2005) 1333-1341.

[22] J. E. Saffitz, A. G. Kleber, Effects of mechanical forces and mediators of hypertrophy on remodeling of gap junctions in the heart, Circ Res. 94 (2004) 585-591.

[23] D. Hakuno, N. Kimura, M. Yoshioka, et al., Periostin advances atherosclerotic and rheumatic cardiac valve degeneration by inducing angiogenesis and MMP production in humans and rodents, J. Clin. Invest. 120(2010) 2292-2306.

[24] G. A. Walker, K. S. Masters, D. N. Shah, et al., Valvular myofibroblast activation by transforming growth factor-beta: implications for pathological extracellular matrix remodeling in heart valve disease, Circ Res. 95 (2004) 253-260.

[25] F. Meier-Stiegen, R. Schwanbeck, K. Bernoth, et al., Activated Notch1 target genes during embryonic cell differentiation depend on the cellular context and include lineage determinants and inhibitors, PLoS One 5 (2010) 1-18.

[26] C. K. Tsang, H. Qi, L. F. Liu, et al., Targeting mammalian target of rapamycin (mTOR) for health and diseases, Drug Discov. Today 12 (2007) 112-124. 
[27] A. J. Muslin, MAPK signalling in cardiovascular health and disease: molecular mechanisms and therapeutic targets, Clin Sci. 115 (2008) 203-218.

[28] J. M. Li, G. Brooks, Cell cycle regulatory molecules (cyclins, cyclin-dependent kinases and cyclin-dependent kinase inhibitors) and the cardiovascular system; potential targets for therapy?, Eur. Heart J. 20 (1999) 406-420. 